Author affiliations appear at the end of this article.

Published online ahead of print at www.jco.org on March 7, 2016

Supported by Merck \& Co, Kenilworth, $\mathrm{NJ}$

Presented at the 50th Annual Meeting of the American Society of Clinical Oncology, Chicago, IL, May 30-June 3, 2014; Society for Immunotherapy of Cancer 2014 Annual Meeting \& Associated Programs, National Harbor MD, November 6-9, 2014; and 51st Annual Meeting of the American Society of Clinical Oncology, Chicago, IL, May 29June 2, 2015

Authors' disclosures of potential conflicts of interest are found in the article online at www.jco.org. Author contributions are found at the end of this article.

Corresponding author: F. Stephen Hodi, MD, Dana-Farber Cancer Institute, 450 Brookline Ave, Boston, MA 02215; e-mail: stephen_hodi@dfci.harvard.edu.

(c) 2016 by American Society of Clinical Oncology

0732-183X/16/3413w-1510w/\$20.00 DOI: 10.1200/JCO.2015.64.0391

\title{
Evaluation of Immune-Related Response Criteria and RECIST v1.1 in Patients With Advanced Melanoma Treated With Pembrolizumab
}

F. Stephen Hodi, Wen-Jen Hwu, Richard Kefford, Jeffrey S. Weber, Adil Daud, Omid Hamid, Amita Patnaik, Antoni Ribas, Caroline Robert, Tara C. Gangadhar, Anthony M. Joshua, Peter Hersey, Roxana Dronca, Richard Joseph, Darcy Hille, Dahai Xue, Xiaoyun Nicole Li, S. Peter Kang, Scot Ebbinghaus, Andrea Perrone, and Jedd D. Wolchok

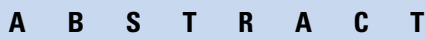

\section{Purpose}

We evaluated atypical response patterns and the relationship between overall survival and best overall response measured per immune-related response criteria (irRC) and Response Evaluation Criteria in Solid Tumors, version 1.1 (RECIST v1.1) in patients with advanced melanoma treated with pembrolizumab in the phase Ib KEYNOTE-001 study (clinical trial information: NCT01295827).

\section{Patients and Methods}

Patients received pembrolizumab 2 or $10 \mathrm{mg} / \mathrm{kg}$ every 2 weeks or every 3 weeks. Atypical responses were identified by using centrally assessed irRC data in patients with $\geq 28$ weeks of imaging. Pseudoprogression was defined as $\geq 25 \%$ increase in tumor burden at week 12 (early) or any assessment after week 12 (delayed) that was not confirmed as progressive disease at next assessment. Response was assessed centrally per irRC and RECIST v1.1.

\section{Results}

Of the 655 patients with melanoma enrolled, 327 had $\geq 28$ weeks of imaging follow-up. Twenty-four $(7 \%)$ of these 327 patients had atypical responses (15 [5\%] with early pseudoprogression and nine [3\%] with delayed pseudoprogression). Of the 592 patients who survived $\geq 12$ weeks, 84 (14\%) experienced progressive disease per RECIST v1.1 but nonprogressive disease per irRC. Two-year overall survival rates were $77.6 \%$ in patients with nonprogressive disease per both criteria $(n=331)$, $37.5 \%$ in patients with progressive disease per RECIST $\vee 1.1$ but nonprogressive disease per irRC $(n=84)$, and $17.3 \%$ in patients with progressive disease per both criteria $(n=177)$.

\section{Conclusion}

Atypical responses were observed in patients with melanoma treated with pembrolizumab. Based on survival analysis, conventional RECIST might underestimate the benefit of pembrolizumab in approximately $15 \%$ of patients; modified criteria that permit treatment beyond initial progression per RECIST v1.1 might prevent premature cessation of treatment.

\section{J Clin Oncol 34:1510-1517. (C) 2016 by American Society of Clinical Oncology}

\section{INTRODUCTION}

Immune checkpoint blockade has emerged as a principal therapeutic modality for the treatment of many cancers. Ipilimumab, a fully human monoclonal antibody that blocks cytotoxic T-lymphocyte-associated protein 4 (CTLA-4), was the first immune checkpoint inhibitor approved by regulatory authorities and prolongs overall survival (OS) in metastatic melanoma. ${ }^{1-3}$ Conventional response criteria might underestimate the therapeutic benefit of immune checkpoint blockade because objective response and prolonged disease stabilization can occur after an initial increase in tumor burden or appearance of new lesions. ${ }^{1,4,5}$ Whereas conventional criteria, such as Response Evaluation Criteria in Solid Tumors (RECIST), were developed based on data from clinical trials of cytotoxic chemotherapy agents for advanced malignancies, ${ }^{6}$ immunerelated response criteria (irRC) were developed to provide more rigorous characterization of the atypical response patterns observed in the phase II development program for ipilimumab in melanoma. ${ }^{1}$ Key differences between irRC $^{1}$ and RECIST 
version $1.1(\mathrm{v} 1.1)^{7}$ are summarized in Table 1. Initial evidence of disease progression is handled differently with irRC compared with conventional response criteria. For example, irRC require confirmation of initial evidence of progressive disease, whereas RECIST do not. Similarly, appearance of new lesions would define progression of disease by RECIST v1.1, whereas new lesions may be added to the sum of the products of the two largest perpendicular diameters of all index lesions at any time point and will only result in progressive disease if the sum is $\geq 25 \%$ compared with nadir. Retrospective evaluations of phase II clinical trials of ipilimumab that included patients with imaging data available beyond initial progression demonstrated that patients who experienced a response or stable disease per irRC had survival rates similar to those of patients who experienced response or stable disease per RECIST. ${ }^{1,8,9}$

Inhibitors of programmed death receptor 1 (PD-1) and one of its ligands, PD-L1, represent the next generation of checkpoint inhibitors that have demonstrated significant anticancer activity. PD- 1 is a surface marker induced on activated T cells ${ }^{10}$; elevated PD-1 expression is a marker for T-cell exhaustion. ${ }^{11}$ Its ligands PD-L1 and PD-L2, normally expressed on antigen-presenting cells and endothelia, can be upregulated on various tumor cells. ${ }^{12}$ Engagement of PD-1 with its ligands leads to inhibition of T-cell receptor signaling ${ }^{13}$ and a lowering of the T-cell apoptotic threshold. ${ }^{14}$ Therefore, tumor cell expression of PD-1 is a clear example of immune surveillance evasion. The PD-1/PD-L1 pathway is likely dominant for tumor escape from effective host immune responses.

Pembrolizumab (MK-3475) is a humanized monoclonal antibody against PD-1 that has been approved in several countries for the treatment of advanced melanoma. US Food and Drug Administration approval of pembrolizumab was based on data obtained from 411 patients enrolled in multiple expansion cohorts of the large KEYNOTE-001 phase I clinical trial. ${ }^{15-17}$ As assessed per RECIST v1.1 by independent central review, the response rate was 39\% in patients with ipilimumab-naive melanoma and 29\% in patients with ipilimumab-treated melanoma. ${ }^{17}$ After an 18 -month median follow-up, $81 \%$ of responders did not experience progressive disease, and the median OS was 25.9 months. ${ }^{17}$
Anecdotal evidence of immune-related response patterns was observed with pembrolizumab during its early clinical development. On the basis of the pembrolizumab mechanism of action and the atypical response patterns observed with ipilimumab, we hypothesized that atypical response patterns would be observed with pembrolizumab and that assessing response per RECIST v1.1 would not provide a comprehensive assessment of the pembrolizumab antitumor effect. By using the larger KEYNOTE-001 655-patient melanoma data set, ${ }^{18}$ we aimed to identify and describe atypical response patterns with pembrolizumab and to assess the relationship between OS and response measured through RECIST v1.1 and irRC.

\section{PATIENTS AND METHODS}

\section{Study Design and Patients}

KEYNOTE-001 (clinical trial information: NCT01295827) was an international, multicenter, open-label, phase Ib study of pembrolizumab for patients with advanced solid tumors, which included multiple melanoma expansion cohorts. Detailed eligibility criteria were published previously. ${ }^{15-18}$ Briefly, adults age 18 years and older with confirmed, unresectable melanoma, an Eastern Cooperative Oncology Group performance status of 0 or 1 , no active infection, no active autoimmune disease or history thereof, no ongoing systemic corticosteroid therapy, and no previous treatment that targeted the PD-1 pathway were included. Both ipilimumab-naive and ipilimumab-treated patients enrolled. The number of previous therapies was unlimited for patients previously treated with ipilimumab and was two or fewer for patients naive to ipilimumab. Patients with active brain metastases or carcinomatous meningitis were excluded.

The study was performed in accordance with protocol, good clinical practice standards, and the Declaration of Helsinki, and protocols and all amendments were approved by the appropriate institutional review board or ethics body at each institution. All patients provided written informed consent.

\section{Treatment and Assessments}

Patients received pembrolizumab intravenously over $30 \mathrm{~min}$ at doses of $2 \mathrm{mg} / \mathrm{kg}$ or $10 \mathrm{mg} / \mathrm{kg}$ every 3 weeks or $10 \mathrm{mg} / \mathrm{kg}$ every 2 weeks. Treatment was continued until confirmed disease progression, intolerable

\begin{tabular}{|c|c|c|}
\hline Category & RECIST v1.1 & irRC \\
\hline Measurement of tumor burden & Unidimensional & Bidimensional \\
\hline Target lesions & Maximum, 5* & Maximum, 15 index lesions \\
\hline New lesion & Results in progressive disease at first appearance & $\begin{array}{l}\text { Up to } 10 \text { new visceral lesions and } 5 \text { cutaneous lesions may be } \\
\text { added to the sum of the products of the two largest } \\
\text { perpendicular diameters of all index lesions at any time point }\end{array}$ \\
\hline Complete response & \multicolumn{2}{|c|}{$\begin{array}{l}\text { Disappearance of all target and nontarget lesions } \\
\text { Nodes must regress to }<10 \mathrm{~mm} \text { short axis } \\
\text { No new lesions } \\
\text { Confirmation required }\end{array}$} \\
\hline Partial response & $\begin{array}{l}\geq 30 \% \text { decrease in tumor burden compared with baseline } \\
\text { Confirmation required }\end{array}$ & $\begin{array}{l}\geq 50 \% \text { decrease in tumor burden compared with baselinet } \\
\text { Confirmation required }\end{array}$ \\
\hline Progressive disease & $\begin{array}{l}\geq 20 \%+5 \text {-mm absolute increase in tumor burden compared } \\
\text { with nadir } \\
\text { Appearance of new lesions or progression of nontarget lesions }\end{array}$ & $\begin{array}{l}\geq 25 \% \text { increase in tumor burden compared with baseline, } \\
\text { nadir, or reset baselinet } \\
\text { New lesions added to tumor burden } \\
\text { Confirmation required }\end{array}$ \\
\hline Stable disease & \multicolumn{2}{|c|}{ Neither partial response nor progressive disease } \\
\hline
\end{tabular}


toxicity, consent withdrawal, physician decision, or any other reason. Radiologic tumor measurements were performed every 12 weeks. Treatment decisions were based on investigator assessment of response per irRC. Per protocol, patients with evidence of radiographic progression could remain on therapy until progression was confirmed on the next imaging assessment performed $\geq 4$ weeks later. Retrospectively, an independent core laboratory (PAREXEL International, Waltham, MA) assessed response per RECIST v1.1 and per irRC. A maximum of 10 target lesions per RECIST v1.1 and 15 index lesions per irRC were assessed per patient. Primary end point assessment was based on RECIST vl.1 by central review.

\section{Analyses}

Atypical responses were identified by using centrally assessed irRC data among patients with measurable disease per irRC and RECIST v1.1 by central review at baseline who were followed by imaging for $\geq 28$ weeks as of the analysis cutoff date of April 18, 2014. The rationale for requiring patients to have $\geq 28$ weeks of follow-up was to allow for two time points after baseline (ie, three total time points by week 28) to identify atypical responses and subsequently to confirm disease progression or response. Early pseudoprogression was defined as $\geq 25 \%$ increase in tumor burden at imaging assessment 1 (week 12) not confirmed as progressive disease per irRC at assessment 2. Delayed pseudoprogression was defined as $\geq 25 \%$ increase in tumor burden at any imaging assessment after the week 12 assessment that was not confirmed as progressive disease per irRC at the next imaging assessment. Patients were excluded from tumor size analysis if they underwent resection or metastasectomy, received subsequent radiation or other therapy, or experienced inflammation at tumor sites. Qualitative assessment of the metastatic sites was performed for patients with atypical responses.

We also evaluated OS in patients with best overall response of stable disease or better per RECIST v1.1 and irRC (first group), versus progressive disease per RECIST v1.1 but nonprogressive disease per irRC (second group), versus progressive disease per RECIST v1.1 and irRC (third group). For all three groups, Kaplan-Meier estimates of OS were assessed. Only patients who survived beyond 12 weeks (ie, the time of the first tumor assessment) were included in this landmark analysis because at least one postbaseline disease assessment was required for patients to qualify for inclusion in the first group.

\section{RESULTS}

\section{Pseudoprogression Analysis}

Of the 655 patients enrolled in the KEYNOTE-001 melanoma expansion cohorts, 327 had $\geq 28$ weeks of imaging follow-up as of April 18, 2014, and were eligible for atypical response analysis. Atypical responses were observed in $24(7.3 \%)$ of 327 patients (15 [4.6\%] with early pseudoprogression and nine [2.8\%] with delayed pseudoprogression; Fig 1). Patterns of atypical response included regression of tumor burden and stable disease per irRC despite the development of new lesions, which would be classified as progressive disease per RECIST, as well as initial increases in the size of target lesions followed by decreases without evidence of new lesions. Atypical responses were observed in both visceral organs and lymph nodes (Fig 2; Appendix Table A1, online only). Among atypical responders, 19 (79\%) were ipilimumab naive (13 early pseudoprogression, six delayed pseudoprogression), four (17\%) were ipilimumab refractory (two each for early and delayed pseudoprogression), and one (4\%) was ipilimumab treated (delayed pseudoprogression). Seven (29\%) had PD-L1-positive tumors, and median baseline tumor size was $52.6 \mathrm{~mm}$ (range, 10.6 to 242.0). These characteristics were similar between patients with early and delayed pseudoprogression (Appendix Table A2, online only). At the time of analysis, all 24 patients who experienced pseudoprogression were alive, with a survival duration ranging from $7.6+$ to $26.4+$ months.

Two examples of early pseudoprogression are shown in Figure 3. In the first, a 56-year-old female with advanced melanoma treated with pembrolizumab $2 \mathrm{mg} / \mathrm{kg}$ every 3 weeks experienced disease progression per RECIST v1.1 and irRC in a skin lesion and liver metastasis at week 12 (Fig 3A). The patient continued pembrolizumab, and at week 24 , both the skin and the liver lesions regressed. By week 24, response per irRC was partial response. Complete response was achieved at week 96 and was ongoing 28 months after enrollment. In the second example, a 72-year-old female with ipilimumab-naive advanced melanoma was treated with pembrolizumab $10 \mathrm{mg} / \mathrm{kg}$ every 2 weeks for two cycles ( 4 weeks). Due to development of grade 2 rash, the patient was switched to a dosage of $10 \mathrm{mg} / \mathrm{kg}$ every 3 weeks and continued therapy with no further dose modification. At the first assessment (week 12), there was a $35.7 \%$ increase in the total tumor burden, but at the follow-up scan performed at week 16, tumor burden decreased by $8.9 \%$ (Fig 3B). As of the last assessment on January 26, 2015 (week 154), the patient remains in partial response by irRC and continues to be on pembrolizumab beyond 3 years with durable partial response.

\section{Comparison of irRC and RECIST v1.1}

The best overall response per irRC by central review for the 15 patients with early pseudoprogression was complete response in three patients, partial response in eight patients, and stable disease in four patients. Per RECIST v1.1, best overall response after initial progression was complete response in three patients, partial response in four patients, stable disease in one patient, and progressive disease in seven patients. The best overall response per irRC by central review for the nine patients with delayed pseudoprogression was complete response in one patient, partial response in two patients, stable disease in five patients, and progressive disease in one patient; per RECIST v1.1, best response was complete response in one patient, partial response in two patients, stable disease in three patients, and progressive disease in three patients. Discrepancies in best overall response were noted for eight patients with early pseudoprogression and two patients with delayed pseudoprogression. Possible factors that contributed to these differences are that irRC uses bidimensional measurements, includes new lesions in the overall tumor burden, and allows for the ability to reset baseline.

As of the analysis cutoff date of April 18, 2014, median followup duration for all 655 patients was 15 months (range, 8 to 29 months). There were 584 patients who had one or more irRC assessments, including 307 (52.6\%) with one or more assessments of progressive disease. For the $92(30.0 \%)$ patients with confirmed progressive disease per irRC after the first progressive disease assessments, median time to the confirmatory measurement was 47 days (range, 20 to 98 days).

Of the 63 patients with $<12$ weeks of observation, 55 died and eight were censored. In the 592 patients who survived $\geq 12$ weeks and as assessed by central review, 331 (56\%) had nonprogressive disease and 177 (30\%) had progressive disease per RECIST v1.1 


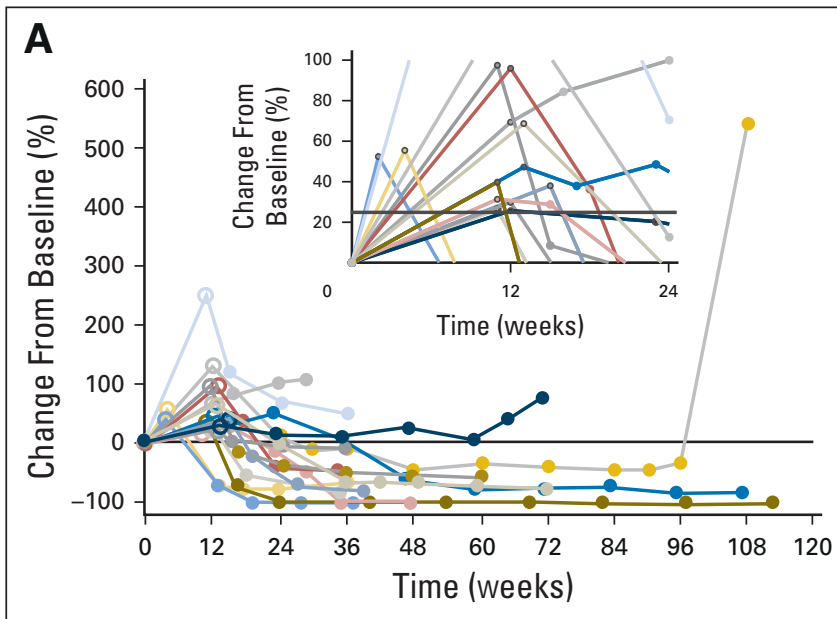

B

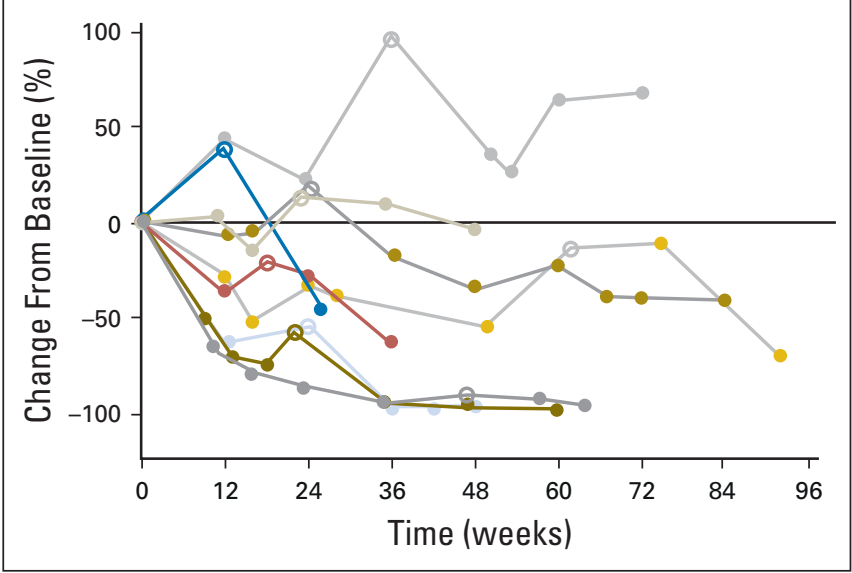

Fig 1. Percent change from baseline in target lesions per immune-related response criteria by central review in patients with early (A) and delayed (B) pseudoprogression. Circles represent times of radiologic assessment. Open circles represent times at which the $25 \%$ threshold was crossed. Colors represent individual patients. The inset in $(\mathrm{A})$ is an enlargement of the change from a baseline of $0 \%$ to $100 \%$ from weeks 0 to 24 , with the $25 \%$ threshold indicated by the horizontal line. In (B), the patient represented by the top gray line did not have a best overall response of progressive disease because progressive disease was not confirmed at the second assessment (change from baseline, $22.1 \%$ ). The patient represented by the dark blue line is considered to have delayed pseudoprogression because a return to nonprogressive disease could not be confirmed at the time of the data cutoff date.

and irRC. A discrepancy in best overall response by central review was observed for the remaining $84(14 \%)$ patients such that best overall response was progressive disease per RECIST v1.1 but nonprogressive disease by irRC. Of these patients, progressive disease per RECIST v1.1 was declared because of a single factor in 59 (70.2\%) patients (Appendix Table A3, online only). In comparison, $88(49.7 \%)$ patients with progressive disease per both criteria had more than one progressive disease factor, including $25(14.1 \%)$ who had $>20 \%$ growth in target lesions, unequivocal growth of nontarget lesions, and appearance of new lesions (Appendix Table A3, online only).

Longitudinal analysis of the change from baseline over time in the sum of target lesions demonstrated that the 84 patients with progressive disease per RECIST v1.1 but nonprogressive disease per irRC were able to gain control of their disease through either stabilization of

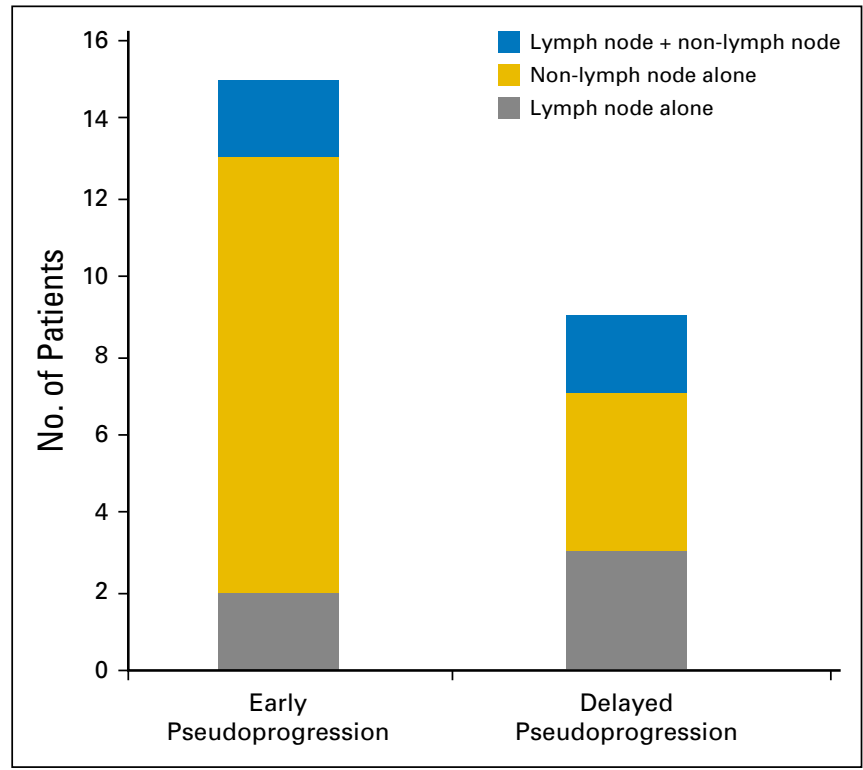

Fig 2. Distribution of target lesions in patients with atypical response patterns.

or a decrease in tumor burden with additional time (Fig 4). OS was longer in the 84 patients with progressive disease by RECIST v1.1 but nonprogressive disease by irRC compared with that in the 177 patients with progressive disease by both RECIST v1.1 and irRC (Fig 5). Median OS was not reached (95\% CI, 25.9 months to not reached) for patients with nonprogressive disease per both criteria, 22.5 months (95\% CI, 16.5 months to not reached) for patients with progressive disease per RECIST v1.1 but nonprogressive disease per irRC, and 8.4 months (95\% CI, 6.6 to 9.9 months) for patients with progressive disease per both criteria. The 2 -year OS rates were $77.6 \%$, $37.5 \%$, and $17.3 \%$, respectively. A general correlation between shorter OS and a higher number of progressive disease criteria was observed, particularly in patients who had progressive disease per RECIST v1.1 and irRC (Appendix Table A3, online only).

\section{DISCUSSION}

Immunotherapeutic agents are being tested as anticancer therapy for many advanced solid tumors and hematologic malignancies. On the basis of the efficacy observed to date, these agents are likely to play a major role in cancer treatment in the near future. Pembrolizumab alone is in clinical development for $>30$ tumor types, including hematologic malignancies, and is approved in several countries for the treatment of advanced melanoma and in the United States, for the treatment of patients with metastatic non-small-cell lung cancer whose tumors express PD-L1 as determined by a Food and Drug Administration-approved test, with disease progression on or after platinum-containing chemotherapy. On the basis of the novel mechanism of action, the likely widespread use of this agent, and the desire to accurately and practically assess clinical benefit, an urgent need exists for new standards for assessing response to pembrolizumab and other novel immunotherapies.

RECIST vl.1, the conventional criteria for tumor measurement, provide a simple, standardized method for defining the therapeutic effect of chemotherapeutic agents. The use of unidimensional tumor 

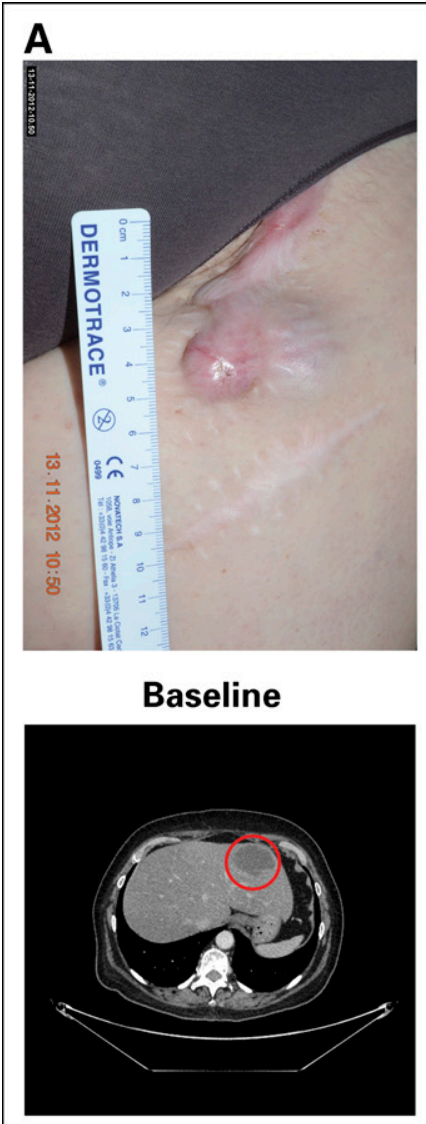

\section{B Baseline}
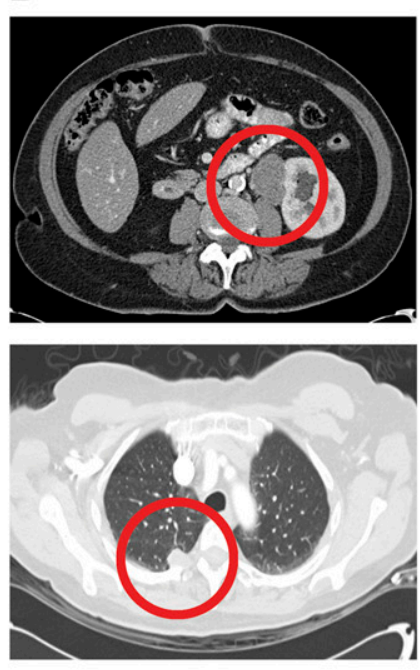

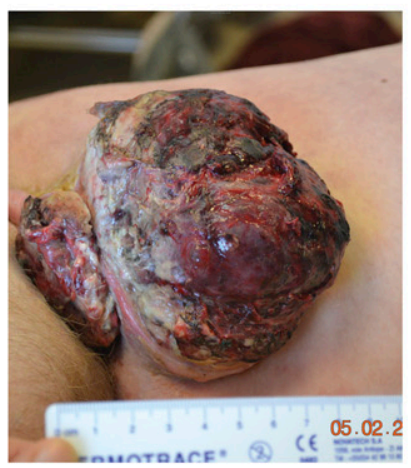

Week 12

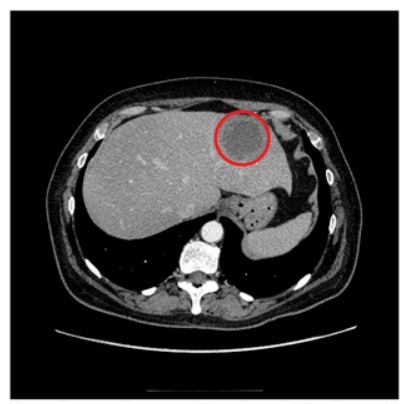

Week 12
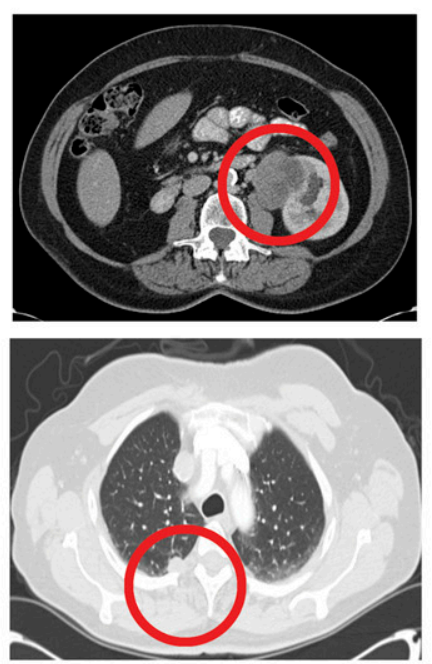

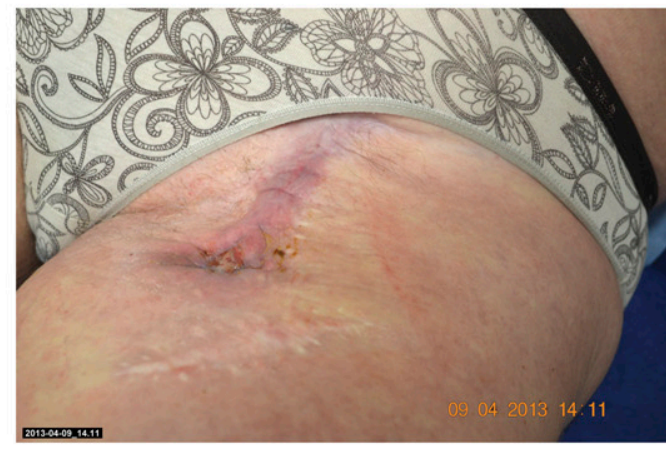

Week 24

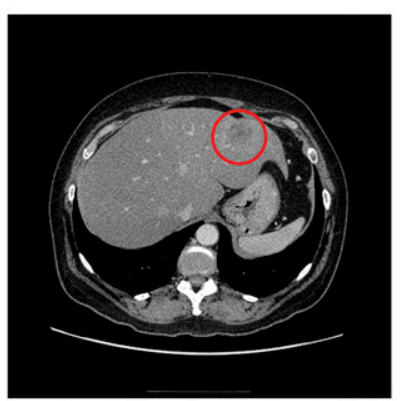

Week 16
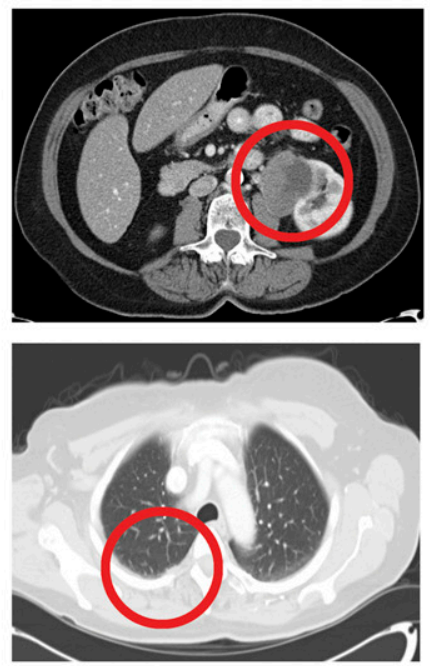

Week 52

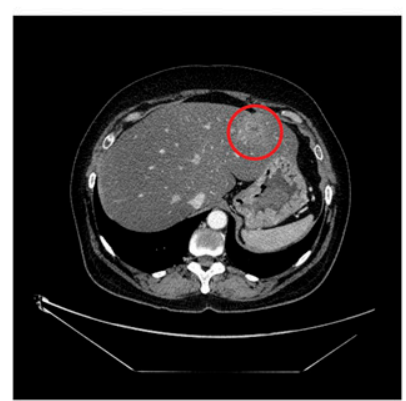

Week 154
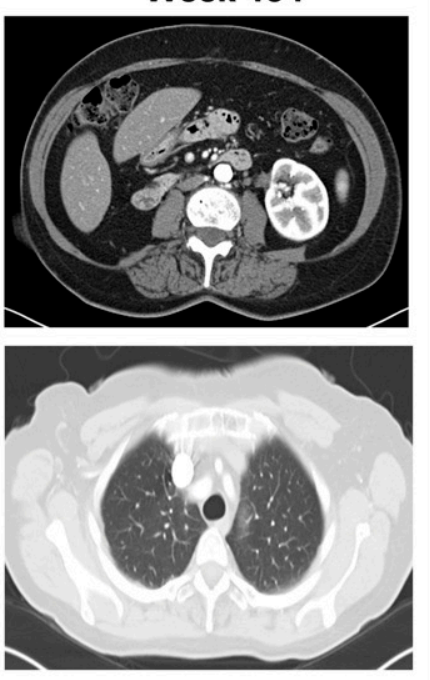

Fig 3. Case studies of patients with early progression. (A) Scans at baseline and 12, 24, and 52 weeks in a 56-year-old woman with advanced melanoma. Per Response

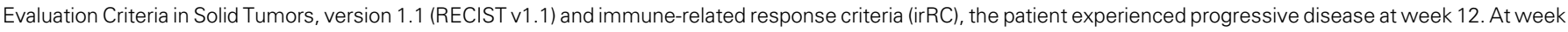

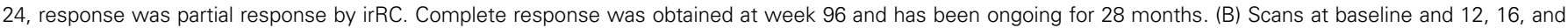

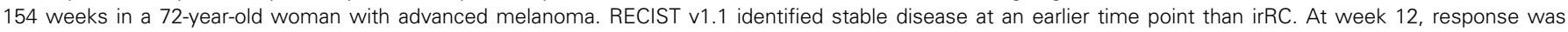

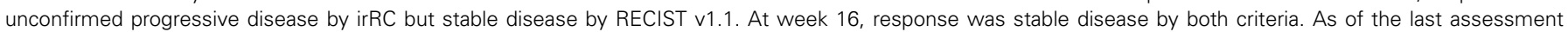
(treatment ongoing), patient remains with partial response.

measurements facilitates their application while minimizing variability, but they are unable to capture responses that occur after disease progression, which might limit their usefulness when assessing response to immunotherapeutic agents. ${ }^{4}$ The irRC were developed to provide standardization for assessing response to immunotherapeutic agents. ${ }^{1}$ Their original conception was based on the modified World Health Organization criteria, which use bidimensional tumor measurements. The irRC incorporate 
A

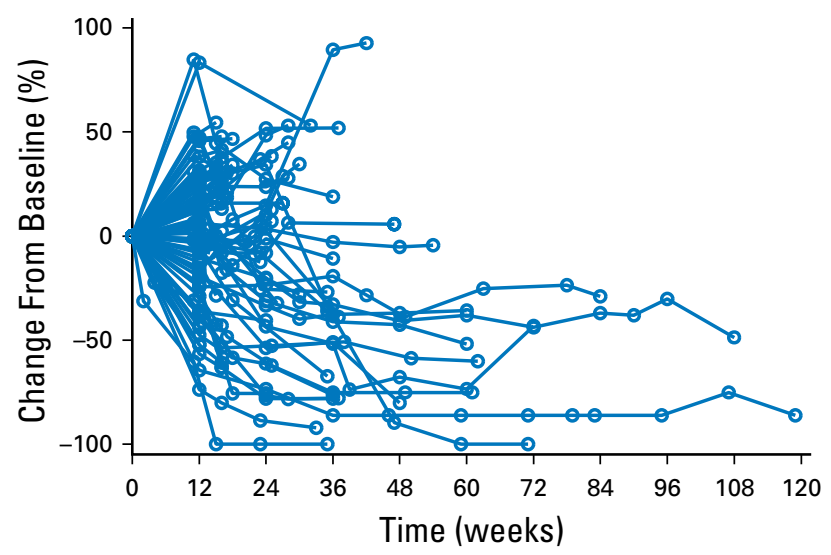

C

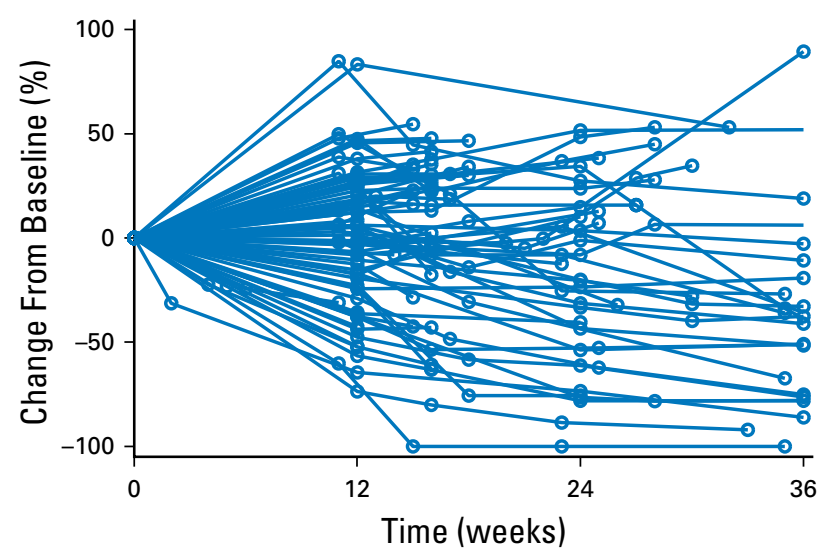

B

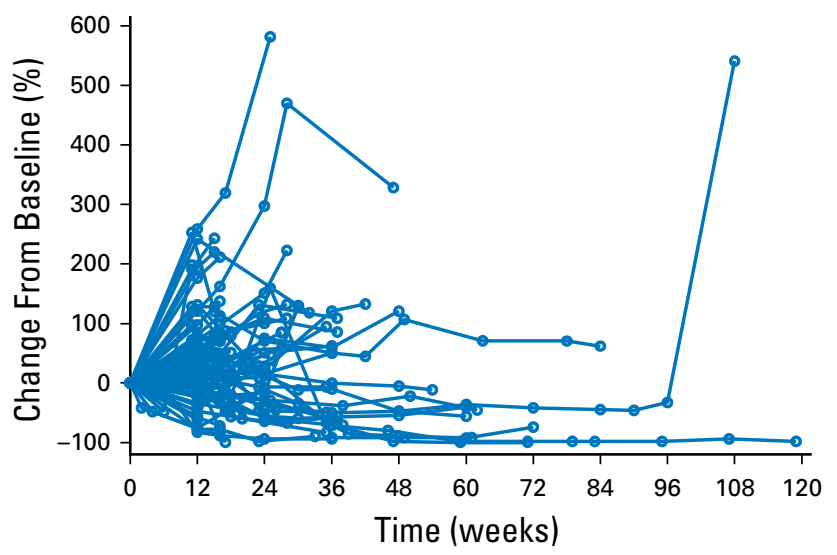

D

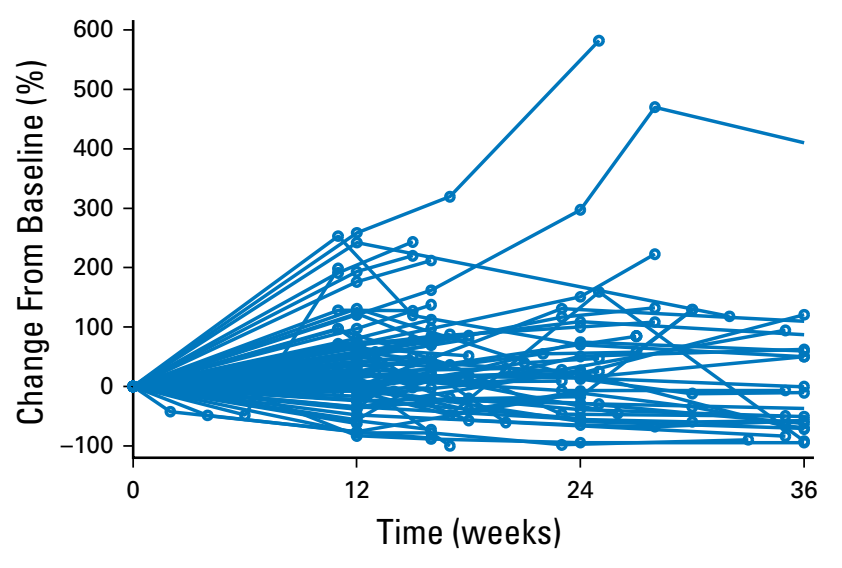

Fig 4. Percent change from baseline in target lesions in patients with best overall response of progressive disease per Response Evaluation Criteria in Solid Tumors,

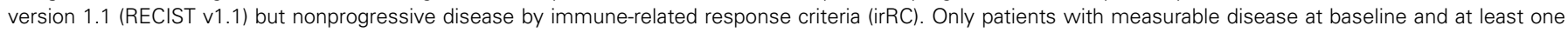

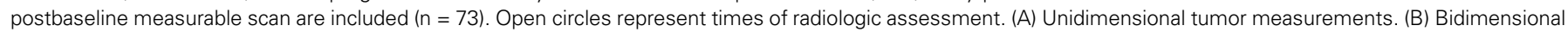

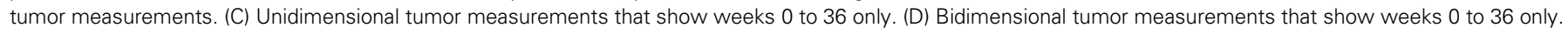

measurable new lesions into the total tumor burden and describe additional patterns of tumor response that can occur after initial increases in tumor burden. ${ }^{4}$ However, greater variability might exist with bidimensional measurements than with unidimensional measurements, ${ }^{5}$ and irRC may not fully capture all patterns of clinical responses. Given the rapid development of effective immuno-oncology agents in multiple cancers, there is a growing effort to develop new standard response criteria for patients treated with immunotherapy to provide for robust clinical end points in evaluating these new treatments. ${ }^{19}$

Similar to observations made with ipilimumab, ${ }^{1}$ we found unique response patterns in certain patients with advanced melanoma treated with the anti-PD-1 antibody pembrolizumab. In the current analysis of patients with melanoma enrolled in KEYNOTE-001, 7\% of evaluable patients experienced early or delayed tumor pseudoprogression. For comparison, in the first report of atypical responses in patients treated with ipilimumab by Wolchok et al, ${ }^{1}$ the incidence was $10 \%$. No clear relationship between PD-L1 expression or prior ipilimumab treatment with pseudoprogression was found. Although relatively infrequent, these unique response patterns have important potential implications for patient management, which is particularly true given observed differences in survival by RECIST v1.1 and irRC per central review. The 84 patients with progressive disease by RECIST v1.1 but nonprogressive disease by irRC had a longer OS than the 177 patients with progressive disease per both criteria, which suggests that RECIST v1.1 might underestimate the benefit of pembrolizumab in approximately $15 \%$ of patients. These data suggest that patients may benefit from receiving treatment beyond initial evidence of radiographic progression and thus support the use of modified response criteria on the basis of immune-related response patterns. Furthermore, clinicians alert to these criteria might be able to avoid otherwise premature termination of potentially effective treatment.

Limitations of the current analysis include the retrospective assessment of response by central review, variability in the patient populations evaluated, subjective assessment by investigators to continue treatment, availability of data for patients who continue treatment beyond progression, and stratification of survival on the basis of postbaseline events. 


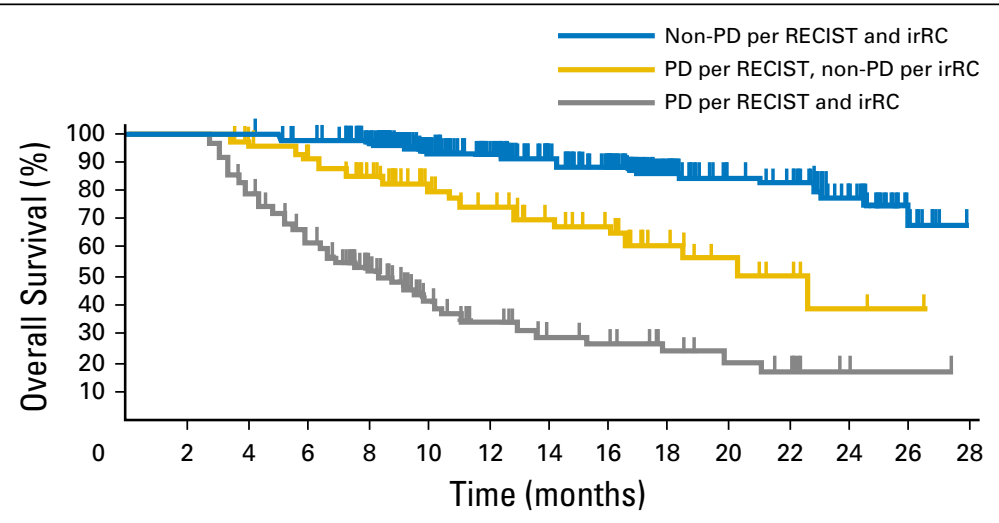

No. at risk

$\begin{array}{lllllllllllllllll}\text { Non-PD per RECIST and irRC } & 331 & 331 & 329 & 321 & 301 & 219 & 192 & 159 & 136 & 79 & 60 & 55 & 31 & 8 & 0\end{array}$ $\begin{array}{lllllllllllllllll}\mathrm{PD} \text { per RECIST, non-PD per irRC } & 84 & 84 & 79 & 71 & 60 & 44 & 37 & 28 & 22 & 13 & 9 & 6 & 3 & 2 & 1\end{array}$ PD per RECIST and irRC

$\begin{array}{ccccccccccccccc}84 & 84 & 79 & 71 & 60 & 44 & 37 & 28 & 22 & 13 & 9 & 6 & 3 & 2 & 1 \\ 177 & 177 & 139 & 109 & 75 & 48 & 33 & 23 & 20 & 15 & 10 & 8 & 1 & 1 & 0\end{array}$

Fig 5. Kaplan-Meier estimates of overall survival on the basis of best overall response per RECIST $v 1.1$ and irRC in patients who survived $\geq 12$ weeks ( $n=592$ ). irRC, immunerelated response criteria; non-PD, nonprogressive disease; PD, progressive disease; RECIST v1.1, Response Evaluation Criteria in Solid Tumors, version 1.1.
Prospective evaluations of irRC and RECIST v1.1 for patients who receive pembrolizumab and other immunotherapeutic agents are needed. Furthermore, the greater awareness of the response patterns witnessed previously for ipilimumab and now with pembrolizumab and other approved and developmental anti-PD-1 and PD-L1 agents ${ }^{20,21}$ has led to growing momentum within the immuno-oncology community to refine imaging criteria. One proposed approach is to modify irRC to follow the same response categories as RECIST and to shift to unidimensional measurements. Alternatively, RECIST could be modified such that after initial evidence of radiologic progression, treatment may be continued until progressive disease is confirmed by imaging performed $>4$ weeks later. New lesions could be effectively followed as nontarget lesions instead of as immediate progressive disease. The details of confirmation of progression could be further delineated based on modeling data. To simplify and standardize these assessments, use of unidimensional measurements and adoption of modified RECIST criteria for immune therapy should also be considered. As a community, we must advocate the sharing of clinical data from multiple studies and immunotherapy agents to greatly hasten and provide rigor to this effort. ${ }^{22}$

\section{AUTHORS' DISCLOSURES OF POTENTIAL CONFLICTS OF INTEREST}

Disclosures provided by the authors are available with this article at www.jco.org.

\section{AUTHOR CONTRIBUTIONS}

Conception and design: F. Stephen Hodi, Wen-Jen Hwu, S. Peter Kang, Scot Ebbinghaus, Andrea Perrone, Jedd D. Wolchok

Provision of study materials or patients: Richard Kefford, Amita Patnaik, Caroline Robert, Tara C. Gangadhar, Anthony M. Joshua, Roxana Dronca Collection and assembly of data: F. Stephen Hodi, Wen-Jen Hwu, Richard Kefford, Omid Hamid, Amita Patnaik, Caroline Robert, Tara C.

Gangadhar, Peter Hersey, Roxana Dronca, Richard Joseph, Xiaoyun Nicole Li, S. Peter Kang, Jedd D. Wolchok

Data analysis and interpretation: F. Stephen Hodi, Richard Kefford, Jeffrey S. Weber, Adil Daud, Omid Hamid, Amita Patnaik, Antoni Ribas, Caroline Robert, Anthony M. Joshua, Roxana Dronca, Richard Joseph, Darcy Hille, Dahai Xue, Xiaoyun Nicole Li, S. Peter Kang, Scot Ebbinghaus, Jedd D. Wolchok

Manuscript writing: All authors

Final approval of manuscript: All authors

\section{REFERENCES}

1. Wolchok JD, Hoos A, O'Day S, et al: Guidelines for the evaluation of immune therapy activity in solid tumors: Immune-related response criteria. Clin Cancer Res 15:7412-7420, 2009

2. Hodi FS, O'Day SJ, McDermott DF, et al: Improved survival with ipilimumab in patients with metastatic melanoma. N Engl J Med 363:711-723, 2010

3. O'Day SJ, Maio M, Chiarion-Sileni $V$, et al: Efficacy and safety of ipilimumab monotherapy in patients with pretreated advanced melanoma: A multicenter single-arm phase II study. Ann Oncol 21: 1712-1717, 2010

4. Nishino M, Jagannathan JP, Krajewski KM, et al: Personalized tumor response assessment in the era of molecular medicine: Cancer-specific and therapy-specific response criteria to complement pitfalls of RECIST. AJR Am J Roentgenol 198: 737-745, 2012

5. Nishino M, Giobbie-Hurder A, Gargano $M$, et al: Developing a common language for tumor response to immunotherapy: Immune-related response criteria using unidimensional measurements. Clin Cancer Res 19:3936-3943, 2013

6. Therasse P, Arbuck SG, Eisenhauer EA, et al: New guidelines to evaluate the response to treatment in solid tumors. J Natl Cancer Inst 92:205-216, 2000

7. Eisenhauer EA, Therasse $P$, Bogaerts $J$, et al: New response evaluation criteria in solid tumours: Revised RECIST guideline (version 1.1). Eur J Cancer 45:228-247, 2009

8. Lynch TJ, Bondarenko I, Luft A, et al: Ipilimumab in combination with paclitaxel and carboplatin as firstline treatment in stage IIIB/IV non-small-cell lung cancer: Results from a randomized, double-blind, multicenter phase II study. J Clin Oncol 30: 2046-2054, 2012

9. Nishino M, Gargano M, Suda M, et al: Optimizing immune-related tumor response assessment: Does reducing the number of lesions impact response assessment in melanoma patients treated with ipilimumab? J Immunother Cancer 2: 17,2014

10. Agata $Y$, Kawasaki $A$, Nishimura $H$, et al: Expression of the PD-1 antigen on the surface of stimulated mouse $T$ and B lymphocytes. Int Immunol 8:765-772, 1996

11. Barber DL, Wherry EJ, Masopust $D$, et al: Restoring function in exhausted CD8 T cells during chronic viral infection. Nature 439:682-687, 2006

12. Keir ME, Butte MJ, Freeman GJ, et al: PD-1 and its ligands in tolerance and immunity. Annu Rev Immunol 26:677-704, 2008 
13. Karwacz K, Bricogne C, MacDonald D, et al: PD-L1 co-stimulation contributes to ligand-induced $\mathrm{T}$ cell receptor down-modulation on CD8+ $\mathrm{T}$ cells. EMBO Mol Med 3:581-592, 2011

14. Dong H, Strome SE, Salomao DR, et al: Tumorassociated $\mathrm{B} 7-\mathrm{H} 1$ promotes T-cell apoptosis: A potential mechanism of immune evasion. Nat Med 8: 793-800, 2002

15. Hamid O, Robert C, Daud A, et al: Safety and tumor responses with lambrolizumab (anti-PD-1) in melanoma. N Engl J Med 369:134-144, 2013

16. Robert $C$, Ribas $A$, Wolchok JD, et al: Antiprogrammed-death-receptor-1 treatment with pembrolizumab in ipilimumab-refractory advanced melanoma: A randomised dose-comparison cohort of a phase 1 trial. Lancet 384:1109-1117, 2014

17. Ribas A, Wolchok JD, Robert C, et al: Updated clinical efficacy of the anti-PD-1 monoclonal antibody pembrolizumab (pembro, MK-3475) in 411 patients (pts) with melanoma (MEL). Pigment Cell Melanoma Res 27:1222-1223, 2014 (abstr)

18. Daud A, Ribas A, Robert $C$, et al: Long-term efficacy of pembrolizumab (pembro; MK-3475) in a pooled analysis of 655 patients (pts) with advanced melanoma (MEL) enrolled in KEYNOTE-001. J Clin Oncol 33: 2015 (suppl; abstr 9005)
19. Bohnsack O, Hoos A, Ludajic K: Adaptation of the immune related response criteria: irRECIST. Ann Oncol 25:iv369, 2014 (suppl 4; abstr 1070P)

20. Robert C, Long GV, Brady B, et al: Nivolumab in previously untreated melanoma without BRAF mutation. N Engl J Med 372:320-330, 2015

21. Herbst RS, Soria J-C, Kowanetz $M$, et al: Predictive correlates of response to the anti-PD-L1 antibody MPDL3280A in cancer patients. Nature 515: 563-567, 2014

22. Chiou VL, Burotto M: Pseudoprogression and immune-related response in solid tumors. J Clin Oncol 33:3541-3543, 2015

\section{Affiliations}

F. Stephen Hodi, Dana-Farber Cancer Institute, Boston, MA; Wen-Jen Hwu, The University of Texas MD Anderson Cancer Center, Houston; Amita Patnaik, South Texas Accelerated Research Therapeutics, San Antonio, TX; Richard Kefford, Westmead Hospital, Melanoma Institute Australia, and Macquarie University; Peter Hersey, University of Sydney, Sydney, Australia; Jeffrey S. Weber, H. Lee Moffitt Cancer Center, Tampa; Richard Joseph, Mayo Clinic, Jacksonville, FL; Adil Daud, University of California San Francisco, San Francisco; Omid Hamid, The Angeles Clinic and Research Institute; Antoni Ribas, University of California Los Angeles, Los Angeles, CA; Caroline Robert, Gustave-Roussy and Paris-Sud University, Villejuif-Paris-Sud, France; Tara C. Gangadhar, Abramson Cancer Center, Philadelphia, PA; Anthony M. Joshua, Princess Margaret Hospital, Toronto, Ontario, Canada; Roxana Dronca, Mayo Clinic, Rochester, MN; Darcy Hille, Dahai Xue, Xiaoyun Nicole Li, S. Peter Kang, Scot Ebbinghaus, and Andrea Perrone, Merck, Kenilworth, NJ; and Jedd D. Wolchok, Memorial Sloan Kettering Cancer Center, New York, NY.

\section{ASCO's New Journal of Global Oncology}

The American Society of Clinical Oncology and David Kerr, MD, DSc, founding Editor-in-Chief of the Society's new Journal of Global Oncology, invite you to submit your next article to JGO.

This new online-only, open access journal fills a growing need for high-quality literature on the array of challenges that health care professionals in resource-limited settings face in caring for patients with cancer and in conducting research. Topics covered include:

- Cancer treatment and diagnosis

- Palliative and supportive care

- Prevention

- Barriers to care

- Epidemiology

- Health policy

Article types that will be considered include original reports, reviews, commentaries, correspondence, special articles, case reports, and editorials.

Submit your article today at GlobalOncologyJournal.org.

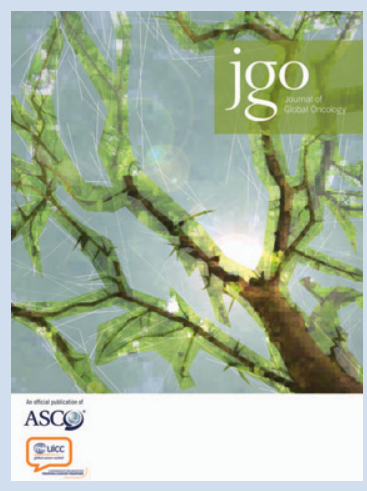


Evaluation of Immune-Related Response Criteria and RECIST v1.1 in Patients With Advanced Melanoma Treated With Pembrolizumab

The following represents disclosure information provided by authors of this manuscript. All relationships are considered compensated. Relationships are self-held unless noted. I = Immediate Family Member, Inst = My Institution. Relationships may not relate to the subject matter of this manuscript. For more information about ASCO's conflict of interest policy, please refer to www.asco.org/rwc or jco.ascopubs.org/site/ifc.

F. Stephen Hodi

Consulting or Advisory Role: Merck, Novartis

Research Funding: Bristol-Myers Squibb (Inst), Merck (Inst), Genentech (Inst), Novartis (Inst)

Patents, Royalties, Other Intellectual Property: Dana-Farber Cancer Institute (Inst)

\section{Wen-Jen Hwu}

Consulting or Advisory Role: Merck

Research Funding: Merck, Bristol-Myers Squibb, GlaxoSmithKline, MedImmune

\section{Richard Kefford}

Honoraria: Merck

Consulting or Advisory Role: GlaxoSmithKline, Roche, Novartis, Merck, Bristol-Myers Squibb, Amgen

Travel, Accommodations, Expenses: Bristol-Myers Squibb, Merck

Jeffrey S. Weber

Stock or Other Ownership: Altor BioScience, Celldex, cCam Biotherapeutics

Honoraria: Bristol-Myers Squibb, Merck, Genentech, AbbVie, AstraZeneca, Daiichi Sankyo, GlaxoSmithKline, Eisai, Altor BioScience, Amgen, Ichor Medical Systems, Celldex, cCam Biotherapeutics, Pieris, Immune Design, Novartis, CytomX Therapeutics, Green Peptide, Medivation

Consulting or Advisory Role: Celldex, cCam Biotherapeutics, Pieris, Altor BioScience, Bristol-Myers Squibb, Merck, Genentech, Amgen,

AstraZeneca, GlaxoSmithKline, AbbVie, Eisai, Green Peptide, Medivation, CytomX Therapeutics, Immune Design

Research Funding: Bristol-Myers Squibb (Inst)

Travel, Accommodations, Expenses: Bristol-Myers Squibb,

GlaxoSmithKline, cCam Biotherapeutics, Merck, AstraZeneca, Genentech, AbbVie, Novartis

Adil Daud

Stock or Other Ownership: OncoSec

Consulting or Advisory Role: Merck, Pfizer, Genentech, Novartis

Research Funding: Merck, Novartis, Pfizer, Bristol-Myers Squibb, OncoSec

\section{Omid Hamid}

Consulting or Advisory Role: Amgen, Genentech, Bristol-Myers Squibb Speakers' Bureau: Genentech, Bristol-Myers Squibb Research Funding: Bristol-Myers Squibb, Genentech, Merck, Merck Serono, Pfizer

Amita Patnaik

Research Funding: Merck (Inst)
Antoni Ribas

Stock or Other Ownership: Kite Pharma

Consulting or Advisory Role: Merck, Amgen, Roche, Pfizer, Millennium Pharmaceuticals

Research Funding: Merck (Inst)

Caroline Robert

Consulting or Advisory Role: Amgen, Merck, Bristol-Myers Squibb, Novartis, Roche

Tara C. Gangadhar

No relationship to disclose

Anthony M. Joshua

No relationship to disclose

\section{Peter Hersey}

No relationship to disclose

\section{Roxana Dronca}

Research Funding: Merck Sharp \& Dohme (Inst)

Richard Joseph

Consulting or Advisory Role: Bristol-Myers Squibb, Nektar Therapeutics, Castle Biosciences

Research Funding: Merck (Inst), Bristol-Myers Squibb (Inst), Genentech (Inst), Amgen (Inst)

\section{Darcy Hille}

Employment: Merck

Stock or Other Ownership: Merck (I)

Dahai Xue

Employment: Merck

Stock or Other Ownership: Merck

Xiaoyun Nicole Li

Employment: Merck

Stock or Other Ownership: Merck

\section{S. Peter Kang}

Employment: Merck

Stock or Other Ownership: Merck

Scot Ebbinghaus

Employment: Merck

Stock or Other Ownership: Merck

Andrea Perrone

Employment: Merck

Stock or Other Ownership: Merck

Jedd D. Wolchok

Consulting or Advisory Role: Bristol-Myers Squibb, Merck, MedImmune, Genentech

Research Funding: Bristol-Myers Squibb (Inst), Merck (Inst) 


\section{Acknowledgment}

Editorial assistance was provided by Tricia Brown and Melanie Leiby (The ApotheCom Merck Oncology Team, Yardley, PA). We thank Linda Gammage (Merck) for providing the computed tomography scans included in the article and Roger Dansey and Yuying Hwang (Merck) for critical review of the manuscript.

\section{Appendix}

\begin{tabular}{|c|c|c|}
\hline Patient No. & Lymph Node & Non-Lymph Node \\
\hline \multicolumn{3}{|c|}{ Early pseudoprogression } \\
\hline 1 & Inguinal & - \\
\hline 2 & - & Peritoneum/omentum \\
\hline 3 & - & Kidney, pleura \\
\hline 4 & Supraclavicular & - \\
\hline 5 & - & Lung \\
\hline 6 & - & Liver \\
\hline 7 & - & Liver, adnexa \\
\hline 8 & - & Lung \\
\hline 9 & - & Peritoneum/omentum, adrenal gland \\
\hline 10 & - & Breast, abdominal wall, chest wall, liver, skin \\
\hline 11 & - & Liver \\
\hline 12 & - & Lung \\
\hline 13 & Axillary & $\begin{array}{l}\text { Adrenal gland, lung, mediastinum, gallbladder, } \\
\text { peritoneum/omentum, retroperitoneum }\end{array}$ \\
\hline 14 & - & Lung \\
\hline 15 & Cervical & Adrenal gland \\
\hline \multicolumn{3}{|c|}{ Delayed pseudoprogression } \\
\hline 1 & Axillary, inguinal & - \\
\hline 2 & - & Adrenal gland \\
\hline 3 & Pelvic & - \\
\hline 4 & - & Liver, peritoneum \\
\hline 5 & - & Kidney \\
\hline 6 & - & Liver, lung \\
\hline 7 & Cervical & - \\
\hline 8 & Axillary & Peritoneum/omentum, retroperitoneum, abdominal wall \\
\hline 9 & Hilar & Peritoneum/omentum \\
\hline
\end{tabular}

Table A2. Key Baseline Characteristics for Patients With Atypical Response

\begin{tabular}{|c|c|c|c|}
\hline & $\begin{array}{l}\text { Early Pseudoprogression } \\
\qquad(\mathrm{n}=15) \text {, No. }(\%)\end{array}$ & $\begin{array}{l}\text { Delayed Pseudoprogression } \\
\qquad(\mathrm{n}=9), \text { No. }(\%)\end{array}$ & $\begin{array}{c}\text { Total } \\
(\mathrm{n}=24), \text { No. }(\%)\end{array}$ \\
\hline \multicolumn{4}{|l|}{ Ipilimumab exposure } \\
\hline Naive & $13(87)$ & $6(67)$ & 19 (79) \\
\hline Refractory & 2 (13) & $2(22)$ & 4 (17) \\
\hline Treated & $0(0)$ & $1(11)$ & $1(4)$ \\
\hline \multicolumn{4}{|l|}{ PD-L1 status } \\
\hline Positive & $4(27)$ & $3(33)$ & $7(29)$ \\
\hline Negative & $2(13)$ & $1(11)$ & $3(13)$ \\
\hline Unknown & $9(60)$ & $5(56)$ & $14(58)$ \\
\hline Baseline tumor size $(\mathrm{mm})$, median (range) & $56.1(10.6-152.1)$ & $49.1(19.6-242.0)$ & $52.6(10.6-242.0)$ \\
\hline
\end{tabular}

Abbreviation: PD-L1, programmed death receptor 1 ligand. 
Hodi et al

Table A3. Overall Survival by Type of Progressive Disease

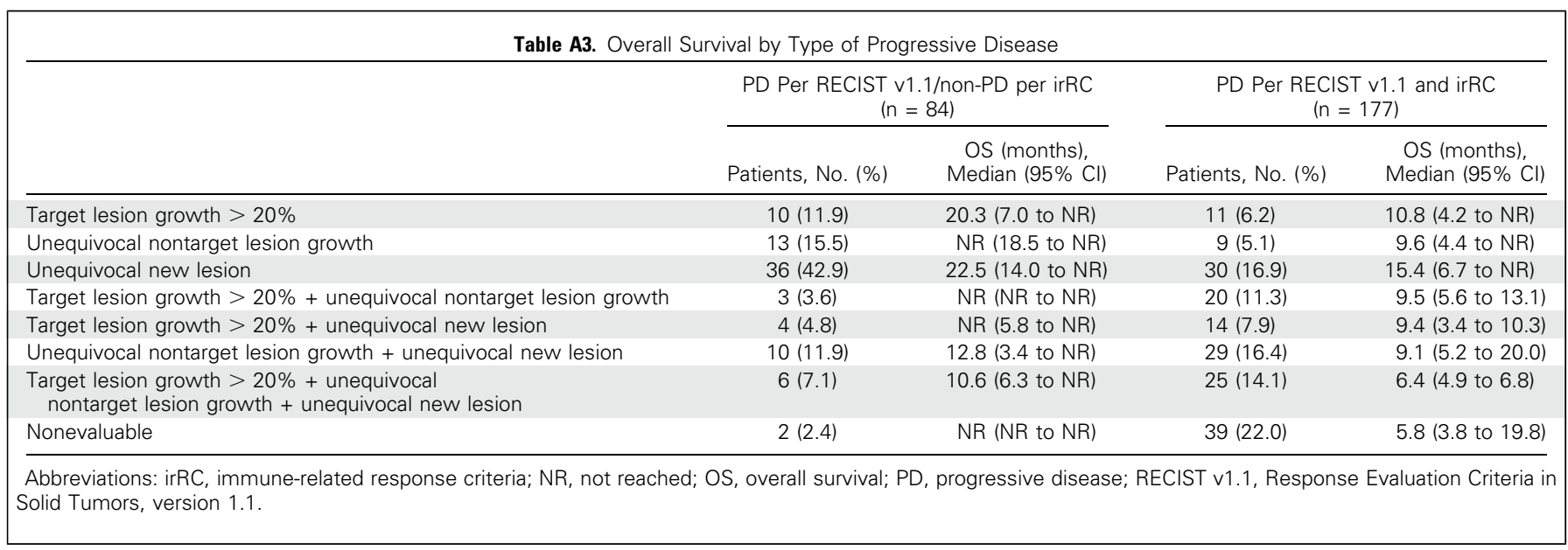

